\title{
Asymptotically Normal Estimators for the Parameters of the Gamma-Exponential Distribution
}

\author{
Alexey Kudryavtsev ${ }^{1,2, *(\mathbb{D})}$ and Oleg Shestakov $1,2,3, *$ (D) \\ 1 Faculty of Computational Mathematics and Cybernetics, M. V. Lomonosov Moscow State University, \\ Moscow 119991, Russia \\ 2 Moscow Center for Fundamental and Applied Mathematics, Moscow 119991, Russia \\ 3 Institute of Informatics Problems, Federal Research Center "Computer Science and Control" of the Russian \\ Academy of Sciences, Moscow 119333, Russia \\ * Correspondence: aakudryavtsev@cs.msu.ru (A.K.); oshestakov@cs.msu.ru (O.S.)
}

Citation: Kudryavtsev, A.; Shestakov, O. Asymptotically Normal Estimators for the Parameters of the Gamma-Exponential Distribution. Mathematics 2021, 9, 273.

https://doi.org/10.3390/math9030273

Academic Editor: Vasile Preda

Received: 24 December 2020

Accepted: 28 January 2021

Published: 30 January 2021

Publisher's Note: MDPI stays neutral with regard to jurisdictional claims in published maps and institutional affiliations.

Copyright: () 2021 by the authors. Licensee MDPI, Basel, Switzerland. This article is an open access article distributed under the terms and conditions of the Creative Commons Attribution (CC BY) license (https: / / creativecommons.org/ licenses/by/4.0/).

\begin{abstract}
Currently, much research attention has focused on generalizations of known mathematical objects in order to obtain adequate models describing real phenomena. An important role in the applied theory of probability and mathematical statistics is the gamma class of distributions, which has proven to be a convenient and effective tool for modeling many real processes. The gamma class is quite wide and includes distributions that have useful properties such as, for example, infinite divisibility and stability, which makes it possible to use distributions from this class as asymptotic approximations in various limit theorems. One of the most important tasks of applied statistics is to obtain estimates of the parameters of the model distribution from the available real data. In this paper, we consider the gamma-exponential distribution, which is a generalization of the distributions from the gamma class. Estimators for some parameters of this distribution are given, and the asymptotic normality of these estimators is proven. When obtaining the estimates, a modified method of moments was used, based on logarithmic moments calculated on the basis of the Mellin transform for the generalized gamma distribution. On the basis of the results obtained, asymptotic confidence intervals for the estimated parameters are constructed. The results of this work can be used in the study of probabilistic models based on continuous distributions with an unbounded non-negative support.
\end{abstract}

Keywords: parameter estimation; gamma-exponential distribution; mixed distributions; generalized gamma distribution; method of moments; asymptotic normality

\section{Introduction}

Estimating unknown parameters is an important problem in applied mathematical statistics. At the same time, in order to improve the consistency of mathematical models and analyzed real processes, researchers consider increasingly complex mathematical abstractions. Many models are traditionally described using continuous distributions with unbounded non-negative supports. For these purposes, special cases of the generalized gamma distribution and beta prime distributions are usually used. The paper considers the problem of estimating the parameters of the distribution proposed in [1], which is closely related to the listed popular distributions.

Definition 1. We say that the random variable $\zeta$ has the gamma-exponential distribution $G E(r, v, s, t, \delta)$ with the parameters of bent $0 \leq r<1$, shape $v \neq 0$, concentration $s, t>0$, and scale $\delta>0$ if its density at $x>0$ is

$$
g_{E}(x)=\frac{|v| x^{t v-1}}{\delta^{t v} \Gamma(s) \Gamma(t)} \mathrm{Ge}_{r, t r+s}\left(-(x / \delta)^{v}\right),
$$


where $E=(r, v, s, t, \delta)$, and $\mathrm{Ge}_{\alpha, \beta}(x)$ is the gamma-exponential function [2]:

$$
\mathrm{Ge}_{\alpha, \beta}(x)=\sum_{k=0}^{\infty} \frac{x^{k}}{k !} \Gamma(\alpha k+\beta), \quad x \in \mathbb{R}, \quad 0 \leq \alpha<1, \quad \beta>0 .
$$

Function (2) generalizes to the case $\beta \neq 1$, the transformation introduced by Le Roy [3] to study generating functions of a special form. In addition, Function (2) can be considered (under some assumptions) as a special case of the Srivastava-Tomovski function [4], that generalizes the Mittag-Leffler function [5].

In [1] it was shown that the distribution (1) adequately describes Bayesian balance models [6]. This is primarily due to the fact that the distribution with the density (1) can be represented as a scaled mixture of two random variables with generalized gamma distributions.

In turn, the generalized gamma distribution $G G(v, q, \theta)$ with the density

$$
f(x)=\frac{|v| x^{v q-1} e^{-(x / \theta)^{v}}}{\theta^{v q} \Gamma(q)}, \quad v \neq 0, \quad q>0, \quad \theta>0, x>0,
$$

proposed in 1925 by the Italian economist L. Amoroso [7] and often associated with E. W. Stacy [8], who considered in 1962 a particular case of the Amoroso distribution, has proven its validity in many applied problems that use continuous distributions with unbounded non-negative support for modeling. The class of distributions (3) is wide enough and includes exponential distribution; $\chi^{2}$-distribution; Erlang distribution; gamma distribution; semi-normal distribution, or distribution of the maximum of the Brownian motion process; Rayleigh distribution; Maxwell-Boltzmann distribution; $\chi$-distribution; Nakagami m-distribution; Wilson-Hilferty distribution; Weibull-Gnedenko distribution and many others, including scaled and inverse analogs of the above.

The problem of estimating parameters of the distribution (3), its special types, and mixtures has a rich history and is still relevant [9-12].

In [1], it was shown that the gamma-exponential distribution has the following properties.

Lemma 1. 1. Let the independent random variables $\lambda$ and $\mu$ have the distributions $G G(v, q, \theta)$ and $G G(u, p, \alpha), u v>0$, respectively. Then the distribution of $\lambda$ coincides with $G E(0, v, \cdot, q, \theta)$; the distribution of $\lambda / \mu$ for $|u|>|v|$ coincides with $G E(v / u, v, p, q, \theta / \alpha)$; the distribution of $\lambda / \mu$ for $|v|>|u|$ coincides with $G E(u / v,-u, q, p, \theta / \alpha)$.

2. For $0<r<1$, the density $g_{E}(x), E=(r, v, s, t, \delta)$, coincides with the density of the ratio of independent random variables with generalized gamma distributions $G G(v, t, \delta)$ and $G G(v / r, s, 1)$.

The possibility of representing the gamma-exponential distribution as a ratio of random variables having the generalized gamma distribution allows it to be used in a wide range of applied problems.

Thus, in demography, the infant mortality rate is defined as the ratio of the number of deaths under the one year age to the number of births over a certain period of time, and the divorce index is defined as the ratio of the total divorce rate to the total marriage rate [13]. In physics, the transformation ratio is the ratio of the output voltage to the input voltage [14], and the universal Kirchhoff function is the ratio of the emissivity to the absorptivity of the body. In queuing theory, the ratio of the intensity of the incoming flow to the intensity of the service determines the system load factor [15]. When simulating emergency situations, the fire hazard of an object is determined by the ratio of the fire threat to the fire protection factor [16]. In reliability theory, the expected uptime is represented as the ratio of the average uptime to the average recovery time [17]. A number of examples can be continued. Each of these characteristics can be considered as the system balance index [6]. The application of a randomized Bayesian approach to the described models 
makes it possible to study the characteristics of the balance index as a scale mixture of probabilistic laws.

In addition, the five-parameter gamma-exponential distribution can be used to model a wide range of real phenomena, due to the wide variety of its possible densities (see Figure 1).

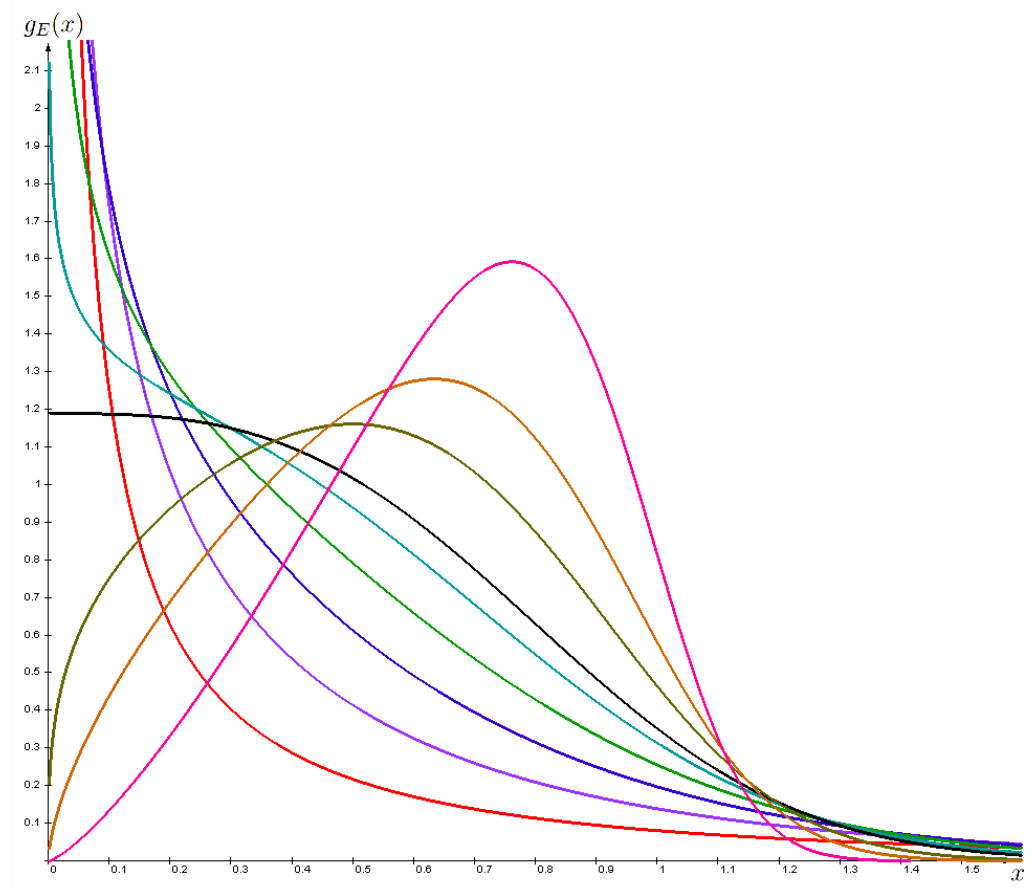

Figure 1. Gamma-exponential distribution densities for different values of parameters.

In practice, the researcher deals with observable quantities that reflect the evolution of the analyzed real process. In relation to these quantities, some model assumptions are made about the form of their distribution. The problem of estimating unknown parameters from real data also arises in the case of modeling a real process using the gamma-exponential distribution. Due to the representation of the density $g_{E}(x)$ in terms of a special gammaexponential function (2), the maximum likelihood method seems to be too complicated. The same can be said about the direct method of moments. For this reason, in [18] it was proposed to estimate the parameters of the gamma-exponential distribution using a modified method based on logarithmic moments.

\section{Estimators for the Parameters of the Gamma-Exponential Distribution}

Let us introduce the estimators for the parameters of the gamma-exponential distribution. To do this, we define the di-gamma function $\psi(z)=\Gamma^{\prime}(z) / \Gamma(z)$ and the functions

$$
\begin{gathered}
R(x)=\sqrt{\frac{v^{2} x-\psi^{\prime}(t)}{\psi^{\prime}(s)}} ; \\
D_{r}(x, y)=\exp \left\{x-\frac{\psi(t)}{v}+\psi(s) \sqrt{\frac{v^{2} y-\psi^{\prime}(t)}{v^{2} \psi^{\prime}(s)}}\right\} ; \\
V(x)=\sqrt[3]{\frac{\psi^{\prime \prime}(t)-r^{3} \psi^{\prime \prime}(s)}{x}} ; \\
D_{v}(x, y)=\exp \left\{x-(\psi(t)-r \psi(s)) \sqrt[3]{\frac{y}{\psi^{\prime \prime}(t)-r^{3} \psi^{\prime \prime}(s)}}\right\} .
\end{gathered}
$$


Let us introduce a notation for the sample logarithmic moments of the random variable $\zeta$ :

$$
L_{k}(X)=\frac{1}{n} \sum_{i=1}^{n} \ln ^{k} X_{i}
$$

where $X=\left(X_{1}, \ldots, X_{n}\right)$ is a sample from the distribution of $\zeta$.

In [18], the following statements were proved.

Lemma 2. For fixed parameters $v$, $t$ and s of the distribution $G E(r, v, s, t, \delta)$ the estimators

$$
\hat{r}(X)=R\left(L_{2}(X)-L_{1}^{2}(X)\right)
$$

and

$$
\hat{\delta}_{r}(X)=D_{r}\left(L_{1}(X), L_{2}(X)-L_{1}^{2}(X)\right)
$$

for the parameters $r$ and $\delta$ are strongly consistent.

Lemma 3. For fixed parameters $r$, $t$ and s of the distribution $G E(r, v, s, t, \delta)$ the estimators

$$
\hat{v}(X)=V\left(L_{3}(X)-3 L_{1}(X) L_{2}(X)+2 L_{1}^{3}(X)\right)
$$

and

$$
\hat{\delta}_{v}(X)=D_{v}\left(L_{1}(X), L_{3}(X)-3 L_{1}(X) L_{2}(X)+2 L_{1}^{3}(X)\right)
$$

for the parameters $v$ and $\delta$ are strongly consistent.

\section{Auxiliary Relations and Statements}

In what follows, we need the derivatives of Functions (4)-(7):

$$
\begin{gathered}
R_{1}(x) \equiv \frac{d R}{d x}(x)=\frac{v^{2}}{2 \psi^{\prime}(s)} \sqrt{\frac{\psi^{\prime}(s)}{v^{2} x-\psi^{\prime}(t)}} ; \\
D_{r, 1}(x, y) \equiv \frac{\partial D_{r}}{\partial x}(x, y)=\exp \left\{x-\frac{\psi(t)}{v}+\psi(s) \sqrt{\frac{v^{2} y-\psi^{\prime}(t)}{v^{2} \psi^{\prime}(s)}}\right\} \\
D_{r, 2}(x, y) \equiv \frac{\partial D_{r}}{\partial y}(x, y)=\frac{\psi(s)}{2 \psi^{\prime}(s)} \sqrt{\frac{v^{2} \psi^{\prime}(s)}{v^{2} y-\psi^{\prime}(t)}} \exp \left\{x-\frac{\psi(t)}{v}+\psi(s) \sqrt{\frac{v^{2} y-\psi^{\prime}(t)}{v^{2} \psi^{\prime}(s)}}\right\} \\
V_{1}(x) \equiv \frac{d V}{d x}(x)=-\frac{\psi^{\prime \prime}(t)-r^{3} \psi^{\prime \prime}(s)}{3 x^{2}} \sqrt[3]{\frac{x^{2}}{\left(\psi^{\prime \prime}(t)-r^{3} \psi^{\prime \prime}(s)\right)^{2}}} ; \\
D_{v, 1}(x, y) \equiv \frac{\partial D_{v}}{\partial x}(x, y)=\exp \left\{x-(\psi(t)-r \psi(s)) \sqrt[3]{\frac{\psi^{\prime \prime}(t)-r^{3} \psi^{\prime \prime}(s)}{y}}\right\} \\
D_{v, 2}(x, y) \equiv \\
\frac{\partial D_{v}}{\partial y}(x, y)=-\frac{\psi(t)-r \psi(s)}{3\left(\psi^{\prime \prime}(t)-r^{3} \psi^{\prime \prime}(s)\right)} \sqrt[3]{\frac{\left(\psi^{\prime \prime}(t)-r^{3} \psi^{\prime \prime}(s)\right)^{2}}{y^{2}}} \times \\
\times \exp \left\{x-(\psi(t)-r \psi(s)) \sqrt[3]{\frac{y}{\psi^{\prime \prime}(t)-r^{3} \psi^{\prime \prime}(s)}}\right\}
\end{gathered}
$$

We also need some moment characteristics of the gamma-exponential distribution (1). Consider the Mellin transform

$$
\mathcal{M}_{\zeta}(z)=\int_{0}^{\infty} x^{z} d F_{\zeta}(x), \quad z \in \mathbb{C} .
$$


We use Lemma 1 and the representation $\zeta \stackrel{d}{=} \lambda / \mu$, where the independent random variables $\lambda$ and $\mu$ have distributions $G G(\nu, t, \delta)$ and $G G(\nu / r, s, 1)$, respectively. Since for $\lambda \sim G G(v, t, \delta)$ the Mellin transform has the form

$$
\mathcal{M}_{\lambda}(z)=\frac{\delta^{z}}{\Gamma(t)} \Gamma\left(t+\frac{z}{v}\right), t+\frac{\operatorname{Re}(z)}{v}>0,
$$

for the ratio of $\lambda \sim G G(\nu, t, \delta)$ to $\mu \sim G G(v / r, s, 1)$

$$
\mathcal{M}_{\lambda / \mu}(z)=\frac{\delta^{z}}{\Gamma(t) \Gamma(s)} \Gamma\left(t+\frac{z}{v}\right) \Gamma\left(s-\frac{r z}{v}\right), t+\frac{\operatorname{Re}(z)}{v}>0, s-\frac{r \operatorname{Re}(z)}{v}>0,
$$

from where we get the form of the characteristic function of the logarithm of $\zeta$ :

$$
\mathrm{E} e^{i y \ln \zeta}=\frac{\delta^{i y}}{\Gamma(t) \Gamma(s)} \Gamma\left(t+\frac{i y}{v}\right) \Gamma\left(s-\frac{i r y}{v}\right), y \in \mathbb{R} .
$$

Differentiating the relation (18) four times, we obtain (the parameters $t$ and $s$ are assumed to be fixed)

$$
\begin{gathered}
\mu_{1}(r, v, \delta) \equiv \mathrm{E} \ln \zeta=\frac{v \ln \delta+\psi(t)-r \psi(s)}{v} ; \\
\mu_{2}(r, v, \delta) \equiv \mathrm{E} \ln ^{2} \zeta=\frac{[v \ln \delta+\psi(t)-r \psi(s)]^{2}}{v^{2}}+\frac{\psi^{\prime}(t)+r^{2} \psi^{\prime}(s)}{v^{2}} ; \\
\mu_{3}(r, v, \delta) \equiv \mathrm{E} \ln ^{3} \zeta=\frac{[v \ln \delta+\psi(t)-r \psi(s)]^{3}}{v^{3}}+ \\
+\frac{3\left(\psi^{\prime}(t)+r^{2} \psi^{\prime}(s)\right)[v \ln \delta+\psi(t)-r \psi(s)]}{v^{3}}+\frac{\psi^{\prime \prime}(t)-r^{3} \psi^{\prime \prime}(s)}{v^{3}} \\
\mu_{4}(r, v, \delta) \equiv \mathrm{E} \ln \zeta=\frac{[v \ln \delta+\psi(t)-r \psi(s)]^{4}}{v^{4}}+ \\
+\frac{6\left(\psi^{\prime}(t)+r^{2} \psi^{\prime}(s)\right)[v \ln \delta+\psi(t)-r \psi(s)]^{2}}{v^{4}}+ \\
+\frac{4\left(\psi^{\prime \prime}(t)-r^{3} \psi^{\prime \prime}(s)\right)[v \ln \delta+\psi(t)-r \psi(s)]}{v^{4}}+ \\
+\frac{3\left(\psi^{\prime}(t)+r^{2} \psi^{\prime}(s)\right)^{2}}{v^{4}}+\frac{\psi^{\prime \prime \prime}(t)+r^{4} \psi^{\prime \prime \prime}(s)}{v^{4}} \\
\sigma_{1}^{2}(r, v) \equiv \mathrm{D} \ln \zeta=\frac{\psi^{\prime}(t)+r^{2} \psi^{\prime}(s)}{v^{2}} ; \\
+\frac{4\left(\psi^{\prime \prime}(t)-r^{3} \psi^{\prime \prime}(s)\right)[v \ln \delta+\psi(t)-r \psi(s)]}{v^{4}}+\frac{2\left(\psi^{\prime}(t)+r^{2} \psi^{\prime}(s)\right)^{2}}{v^{4}}+\frac{\psi^{\prime \prime \prime}(t)+r^{4} \psi^{\prime \prime \prime}(s)}{v^{4}}
\end{gathered}
$$

Further arguments are based on the following statements [19].

Lemma 4. In $\mathbb{R}^{n}$, the random vector $X_{n}$ converges in distribution to the random vector $X$ if and only if each linear combination of the components of $X_{n}$ converges in distribution to the same linear combination of the components of $X$.

Lemma 5. Suppose that in $\mathbb{R}^{k}$

$$
\sqrt{n}\left(T_{n 1}, \ldots, T_{n k}\right) \Longrightarrow N(\mu, \Sigma), n \rightarrow \infty,
$$


with $\Sigma$ a covariance matrix. Let $g(t)=g\left(t_{1}, \ldots, t_{k}\right)$ be a real-valued function with a nonzero differential at $t=\mu$. Put

$$
d=\left(\left.\frac{\partial g}{\partial t_{1}}\right|_{t=\mu}, \ldots,\left.\frac{\partial g}{\partial t_{k}}\right|_{t=\mu}\right) .
$$

Then $\sqrt{n} g\left(T_{n 1}, \ldots, T_{n k}\right) \Longrightarrow N\left(g(\mu), d \Sigma d^{T}\right)$.

\section{Asymptotic Normality of the Estimators for the Parameters of the Gamma-Exponential Distribution}

Let us formulate the statements about the asymptotic normality of the estimators (8)-(11). Let us fix the parameters of shape $v$ and concentration $t$ and $s$. The following statements hold.

Theorem 1. The estimator (8) for the unknown parameter $r$ is asymptotically normal:

$$
\sqrt{n} \frac{\hat{r}(X)-r}{\sqrt{\frac{v^{4} \sigma_{2}^{2}(r, v, \delta)}{4 r^{2}\left(\psi^{\prime}(s)\right)^{2}}}} \Longrightarrow N(0,1), n \rightarrow \infty,
$$

where $\sigma_{2}^{2}(r, v, \delta)$ is given by (24).

Proof of Theorem 1. The statistic $L_{2}(X)-L_{1}^{2}(X)$ is a sample logarithmic variance that is representable as a sum of independent identically distributed random variables and has the mean $\sigma_{1}^{2}(r, v)$ and the variance $\sigma_{2}^{2}(r, v, \delta) / n$. Thus, when $n \rightarrow \infty$

$$
\sqrt{n} \frac{L_{2}(X)-L_{1}^{2}(X)-\sigma_{1}^{2}(r, v)}{\sqrt{\sigma_{2}^{2}(r, v, \delta)}} \Longrightarrow N(0,1),
$$

where $\sigma_{1}^{2}(r, v)$ is defined in (23). In addition, at the point $x=\sigma_{1}^{2}(r, v)$ the function $R(x)$, defined in (4), has a nonzero derivative $R_{1}(x)$, defined in (12).

Next, we use Lemma 5. Since in this case we consider a one-dimensional space $(k=1)$, in terms of the notation of Lemma 5

$$
d \Sigma d^{T}=\left(R_{1}\left(\sigma_{1}^{2}(r, v)\right)\right)^{2} \sigma_{2}^{2}(r, v, \delta),
$$

and it follows that

$$
\sqrt{n} \frac{R\left(L_{2}(X)-L_{1}^{2}(X)\right)-R\left(\sigma_{1}^{2}(r, v)\right)}{\sqrt{\left(R_{1}\left(\sigma_{1}^{2}(r, v)\right)\right)^{2} \sigma_{2}^{2}(r, v, \delta)}} \Longrightarrow N(0,1),
$$

which concludes the proof.

Repeating the reasoning from [19], it can be shown that Lemma 2 and the continuity of the function (24) in $r$ and $\delta$ and the function (12) at $x=\sigma_{1}^{2}(r, v)$ imply the following statement.

Corollary 1. When $n \rightarrow \infty$

$$
\sqrt{n} \frac{\hat{r}(X)-r}{\sqrt{\left(R_{1}\left(L_{2}(X)-L_{1}^{2}(X)\right)\right)^{2} \sigma_{2}^{2}\left(\hat{r}(X), v, \hat{\delta}_{r}(X)\right)}} \Longrightarrow N(0,1),
$$

where the functions $R_{1}$ and $\sigma_{2}^{2}$ are given by the relations (12) and (24), and the statistics $\hat{r}(X)$ and $\hat{\delta}_{r}(X)$ are defined in (8) and (9). 
Consider the estimator for the parameter $\delta$ with fixed parameters $v, t$ and $s$. Let us introduce the notation

$$
\begin{gathered}
\sigma_{12}(r, v)=\frac{\psi^{\prime \prime}(t)-r^{3} \psi^{\prime \prime}(s)}{v^{3}} \\
s_{1}^{2}(r, v, \delta)=\delta^{2} \sigma_{1}^{2}(r, v)+\frac{v \delta^{2} \psi(s) \sigma_{12}(r, v)}{r \psi^{\prime}(s)}+\frac{v^{2} \delta^{2} \psi^{2}(s) \sigma_{2}^{2}(r, v, \delta)}{4 r^{2}\left(\psi^{\prime}(s)\right)^{2}}
\end{gathered}
$$

where $\sigma_{1}^{2}(r, v), \sigma_{2}^{2}(r, v, \delta)$ and $\sigma_{12}(r, v)$ are given by the relations (23), (24) and (26), respectively.

Theorem 2. The estimator (9) for the unknown parameter $\delta$ is asymptotically normal:

$$
\sqrt{n} \frac{\hat{\delta}_{r}(X)-\delta}{\sqrt{s_{1}^{2}(r, v, \delta)}} \Longrightarrow N(0,1), n \rightarrow \infty,
$$

where $s_{1}^{2}(r, v, \delta)$ is given by (27).

Proof of Theorem 2. Note that $L_{1}(x)$ is the sum of independent identically distributed random variables, therefore

$$
\sqrt{n} \frac{L_{1}(X)-\mu_{1}(r, v, \delta)}{\sqrt{\sigma_{1}^{2}(r, v)}} \Longrightarrow N(0,1), \quad n \rightarrow \infty,
$$

where $\mu_{1}(r, v, \delta)$ and $\sigma_{1}^{2}(r, v)$ are given by (19) and (23). The statisitcs $L_{2}(X)-L_{1}^{2}(X)$ is also asymptotically normal, and (25) holds. Consider the covariance matrix

$$
\Sigma=\left(\begin{array}{cc}
\sigma_{1}^{2}(r, v) & \sigma_{12}(r, v) \\
\sigma_{12}(r, v) & \sigma_{2}^{2}(r, v, \delta)
\end{array}\right)
$$

Note that

$$
\operatorname{cov}\left(L_{1}(X), L_{2}(X)-L_{1}^{2}(X)\right)=\frac{n-1}{n^{2}} \sigma_{12}(r, v)=\frac{1}{n} \sigma_{12}(r, v)+o\left(n^{-1}\right), \quad n \rightarrow \infty .
$$

The statistics $L_{1}(X)$ and $L_{2}(X)-L_{1}^{2}(X)$ together with any of their linear combinations have the property of asymptotic normality with corresponding limit means depending on $\left(\mu_{1}(r, v, \delta), \sigma_{1}^{2}(r, v)\right)$, and variances defined by the matrix $\Sigma$. Therefore, Lemma 4 implies the convergence of vectors

$$
\sqrt{n}\left(L_{1}(X), L_{2}(X)-L_{1}^{2}(X)\right) \Longrightarrow N\left(\left(\mu_{1}(r, v, \delta), \sigma_{1}^{2}(r, v)\right), \Sigma\right) .
$$

In addition, the partial derivatives $D_{r, 1}(x, y)$ and $D_{r, 2}(x, y)$, defined in (13) and (14), of the function $D_{r}(x, y)$, defined in (5), are nonzero at $(x, y)=\left(\mu_{1}(r, v, \delta), \sigma_{1}^{2}(r, v)\right)$. Hence, Lemma 5 implies the convergence

$$
\sqrt{n} D_{r}\left(L_{1}(X), L_{2}(X)-L_{1}^{2}(X)\right) \Longrightarrow N\left(D_{r}\left(\mu_{1}(r, v, \delta), \sigma_{1}^{2}(r, v)\right), d \Sigma d^{T}\right),
$$

where

$$
d=\left(D_{r, 1}\left(\mu_{1}(r, v, \delta), \sigma_{1}^{2}(r, v)\right), D_{r, 2}\left(\mu_{1}(r, v, \delta), \sigma_{1}^{2}(r, v)\right)\right) .
$$

The relation

$$
d \Sigma d^{T}=\delta^{2} \sigma_{1}^{2}(r, v)+\frac{v \delta^{2} \psi(s)}{r \psi^{\prime}(s)} \sigma_{12}(r, v)+\frac{v^{2} \delta^{2} \psi^{2}(s)}{4 r^{2}\left(\psi^{\prime}(s)\right)^{2}} \sigma_{2}^{2}(r, v, \delta)
$$

concludes the proof. 
Lemma 2 and the continuity of the function (27) in $r$ and $\delta$ and functions (13) and (14) at $(x, y)=\left(\mu_{1}(r, v, \delta), \sigma_{1}^{2}(r, v)\right)$ imply the following statement.

Corollary 2. When $n \rightarrow \infty$

$$
\sqrt{n} \frac{\hat{\delta}_{r}(X)-\delta}{\sqrt{s_{1}^{2}\left(\hat{r}(X), v, \hat{\delta}_{r}(X)\right)}} \Longrightarrow N(0,1),
$$

where the function $s_{1}^{2}$ is given by (27), and the statistics $\hat{r}(X)$ and $\hat{\delta}_{r}(X)$ are defined in (8) and (9).

Let us fix the parameters $r, t$ and $s$, and introduce the notation

$$
\sigma_{3}^{2}(r, v, \delta) \equiv \mathrm{D} \ln ^{3} \zeta
$$

Remark 1. The analytical form of the variance in (29) is obtained similarly to (23) and (24) by differentiating the characteristic function of the random variable $\ln \zeta$. The explicit form of this expression is not given due to its cumbersomeness.

Theorem 3. The estimator (10) for the unknown parameter $v$ is asymptotically normal:

$$
\sqrt{n} \frac{\hat{v}(X)-v}{\sqrt{\frac{v^{8} \sigma_{3}^{2}(r, v, \delta)}{9\left(\psi^{\prime \prime}(t)-r^{3} \psi^{\prime \prime}(s)\right)^{2}}}} \Longrightarrow N(0,1), n \rightarrow \infty,
$$

where $\sigma_{3}^{2}(r, v, \delta)$ is given by (29).

Proof of Theorem 3. Based on the form of statistics $L_{k}(X)$ as sums of random variables, we obtain

$$
\sqrt{n} \frac{L_{3}(X)-3 L_{1}(X) L_{2}(X)+2 L_{1}^{3}(X)-\sigma_{12}(r, v)}{\sqrt{\sigma_{3}^{2}(r, v, \delta)}} \Longrightarrow N(0,1), n \rightarrow \infty,
$$

since

$$
\mathrm{E}\left(L_{3}(X)-3 L_{1}(X) L_{2}(X)+2 L_{1}^{3}(X)\right)=\frac{n^{2}-3 n+2}{n^{2}} \cdot \frac{\psi^{\prime \prime}(t)-r^{3} \psi^{\prime \prime}(s)}{v^{3}}=\sigma_{12}(r, v)+o(1)
$$

and

$$
\mathrm{D}\left(\ln ^{3} X_{1}-3 \ln X_{1} L_{2}(X)+2 \ln X_{1} L_{1}^{2}(X)\right)=\sigma_{3}^{2}(r, v, \delta)+o(1),
$$

which obviously follows from the inequality

$$
(\sqrt{\mathrm{D} \xi}-\sqrt{\mathrm{D} \eta})^{2} \leq \mathrm{D}(\xi+\eta) \leq(\sqrt{\mathrm{D} \xi}+\sqrt{\mathrm{D} \eta})^{2},
$$

valid for any random variables $\xi$ and $\eta$ with finite variances. In addition, at the point $x=\sigma_{12}(r, v)$ the function $V(x)$, defined in (6), has a nonzero derivative $V_{1}(x)$, defined in (15).

In terms of Lemma 5 for the one-dimensional case we obtain the relation

$$
d \Sigma d^{T}=\left(V_{1}\left(\sigma_{12}(r, v)\right)\right)^{2} \sigma_{3}^{2}(r, v, \delta),
$$

from which it follows that

$$
\sqrt{n} \frac{V\left(L_{3}(X)-3 L_{1}(X) L_{2}(X)+2 L_{1}^{3}(X)\right)-V\left(\sigma_{12}(r, v)\right)}{\sqrt{\left(V_{1}\left(\sigma_{12}(r, v)\right)\right)^{2} \sigma_{3}^{2}(r, v, \delta)}} \Longrightarrow N(0,1) .
$$


This concludes the proof.

Lemma 3 and the continuity of the function (29) in $v$ and $\delta$ and the function (15) at $x=\sigma_{12}(r, v)$ imply the following statement.

Corollary 3. When $n \rightarrow \infty$

$$
\sqrt{n} \frac{\hat{v}(X)-v}{\sqrt{\left(V_{1}\left(L_{3}(X)-3 L_{1}(X) L_{2}(X)+2 L_{1}^{3}(X)\right)\right)^{2} \sigma_{3}^{2}\left(r, \hat{v}(X), \hat{\delta}_{v}(X)\right)}} \Longrightarrow N(0,1),
$$

where the functions $V_{1}$ and $\sigma_{3}^{2}$ are given by (15) and (29), and the statistics $\hat{v}(X)$ and $\hat{\delta}_{v}(X)$ are defined in (10) and (11).

Consider the estimator for the parameter $\delta$ with fixed parameters $r, t$ and $s$. Let us introduce the notation

$$
\begin{gathered}
s_{12}(r, v, \delta)=\frac{\psi^{\prime \prime \prime}(t)-6 \psi^{\prime}(t)[v \ln \delta+\psi(t)-r \psi(s)]^{2}}{v^{4}}+ \\
+\frac{r^{4} \psi^{\prime \prime \prime}(s)-6 r^{2} \psi^{\prime}(s)[v \ln \delta+\psi(t)-r \psi(s)]^{2}}{v^{4}} ; \\
s_{2}^{2}(r, v, \delta)=\delta^{2} \sigma_{1}^{2}(r, v)-\frac{2 v^{2} \delta^{2}(\psi(t)-r \psi(s)) s_{12}(r, v, \delta)}{3\left(\psi^{\prime \prime}(t)-r^{3} \psi^{\prime \prime}(s)\right)}+\frac{v^{4} \delta^{2}(\psi(t)-r \psi(s))^{2} \sigma_{3}^{2}(r, v, \delta)}{9\left(\psi^{\prime \prime}(t)-r^{3} \psi^{\prime \prime}(s)\right)^{2}},
\end{gathered}
$$

where $\sigma_{1}^{2}(r, v), \sigma_{3}^{2}(r, v, \delta)$ and $s_{12}(r, v)$ are given by (23), (29) and (32), respectively.

Theorem 4. The estimator (11) for the unknown parameter $\delta$ is asymptotically normal:

$$
\sqrt{n} \frac{\hat{\delta}_{v}(X)-\delta}{\sqrt{s_{2}^{2}(r, v, \delta)}} \Longrightarrow N(0,1), n \rightarrow \infty,
$$

where $s_{2}^{2}(r, v, \delta)$ is given by (33).

Proof of Theorem 4. Note that when $n \rightarrow \infty$ the relations (28) and (31) hold. Consider the covariance matrix

$$
\Sigma=\left(\begin{array}{cc}
\sigma_{1}^{2}(r, v) & s_{12}(r, v, \delta) \\
s_{12}(r, v, \delta) & \sigma_{3}^{2}(r, v, \delta)
\end{array}\right)
$$

Since

$$
\begin{gathered}
\operatorname{cov}\left(L_{1}(X), L_{3}(X)-3 L_{1}(X) L_{2}(X)+2 L_{1}^{3}(X)\right)= \\
=\frac{\mu_{4}(r, v, \delta)}{n}-\frac{4 \mu_{3}(r, v, \delta) \mu_{1}(r, v, \delta)}{n}-\frac{3 \mu_{2}^{2}(r, v, \delta)}{n}+ \\
+\frac{4 \mu_{2}(r, v, \delta) \mu_{1}^{2}(r, v, \delta)}{n}+\frac{2 \mu_{1}^{4}(r, v, \delta)}{n}+2 \mathrm{D} L_{1}^{2}(X)+o\left(n^{-1}\right), n \rightarrow \infty,
\end{gathered}
$$

and

$$
\begin{gathered}
n^{4} \mathrm{E} L_{1}^{4}(X)=n \mu_{4}(r, v, \delta)+4 n(n-1) \mu_{3}(r, v, \delta) \mu_{1}(r, v, \delta)+ \\
+3 n(n-1) \mu_{2}^{2}(r, v, \delta)+3 n(n-1)(n-2) \mu_{2}(r, v, \delta) \mu_{1}^{2}(r, v, \delta)+ \\
+\left(n^{4}-3 n(n-1)(n-2)-7 n(n-1)-n\right) \mu_{1}^{4}(r, v, \delta),
\end{gathered}
$$

where the moments $\mu_{k}(r, v, \delta), k=1, \ldots, 4$, are given by (19)-(22),

$$
\operatorname{cov}\left(L_{1}(X), L_{3}(X)-3 L_{1}(X) L_{2}(X)+2 L_{1}^{3}(X)\right)=\frac{1}{n} s_{12}(r, v, \delta)+o\left(n^{-1}\right) .
$$


The statistics $L_{1}(X)$ and $L_{3}(X)-3 L_{1}(X) L_{2}(X)+2 L_{1}^{3}(X)$ together with any of their linear combinations have the property of asymptotic normality with corresponding limit means depending on $\left(\mu_{1}(r, v, \delta), \sigma_{12}(r, v)\right)$, and variances defined by the matrix $\Sigma$. Therefore, Lemma 4 implies the convergence of vectors

$$
\sqrt{n}\left(L_{1}(X), L_{3}(X)-3 L_{1}(X) L_{2}(X)+2 L_{1}^{3}(X)\right) \Longrightarrow N\left(\left(\mu_{1}(r, v, \delta), \sigma_{12}(r, v)\right), \Sigma\right),
$$

where $\mu_{1}(r, v, \delta)$ and $\sigma_{12}(r, v)$ are defined in (19) and (26). In addition, the partial derivatives $D_{v, 1}(x, y)$ and $D_{v, 2}(x, y)$, defined in (16) and (17) of the function $D_{v}(x, y)$, defined in (7), are nonzero at $(x, y)=\left(\mu_{1}(r, v, \delta), \sigma_{12}(r, v)\right)$. Hence, by Lemma 5

$$
\sqrt{n} D_{v}\left(L_{1}(X), L_{3}(X)-3 L_{1}(X) L_{2}(X)+2 L_{1}^{3}(X)\right) \Longrightarrow N\left(D_{v}\left(\mu_{1}(r, v, \delta), \sigma_{12}(r, v)\right), d \Sigma d^{T}\right),
$$

where

$$
d=\left(D_{v, 1}\left(\mu_{1}(r, v, \delta), \sigma_{12}(r, v)\right), D_{v, 2}\left(\mu_{1}(r, v, \delta), \sigma_{12}(r, v)\right)\right) .
$$

The realtion

$$
d \Sigma d^{T}=\delta^{2} \sigma_{1}^{2}(r, v)-\frac{2 v^{2} \delta^{2}(\psi(t)-r \psi(s)) s_{12}(r, v, \delta)}{3\left(\psi^{\prime \prime}(t)-r^{3} \psi^{\prime \prime}(s)\right)}+\frac{v^{4} \delta^{2}(\psi(t)-r \psi(s))^{2} \sigma_{3}^{2}(r, v, \delta)}{9\left(\psi^{\prime \prime}(t)-r^{3} \psi^{\prime \prime}(s)\right)^{2}}
$$

concludes the proof.

Lemma 3 and the continuity of the function (33) in $v$ and $\delta$ and the functions (16) and (17) at $(x, y)=\left(\mu_{1}(r, v, \delta), \sigma_{12}(r, v)\right)$ imply the following statement.

Corollary 4. When $n \rightarrow \infty$

$$
\sqrt{n} \frac{\hat{\delta}_{v}(X)-\delta}{\sqrt{s_{2}^{2}\left(r, \hat{v}(X), \hat{\delta}_{v}(X)\right)}} \Longrightarrow N(0,1),
$$

where the function $s_{2}^{2}$ is given by (33), and the statistics $\hat{v}(X)$ and $\hat{\delta}_{v}(X)$ are defined in (10) and (11).

\section{Confidence Intervals}

On the basis of Corollaries 1-4, asymptotic confidence intervals for unknown parameters of the gamma-exponential distribution can be constructed.

By $u_{\gamma}$ we denote the $(1+\gamma) / 2$-quantile of the standard normal distribution.

Corollary 5. The asymptotic confidence interval with the confidence level $\gamma$ based on the estimator (8) for the unknown parameter $r$ has the form

$$
\left(S_{r}(X), T_{r}(X)\right)=\left(\hat{r}(X)-\frac{u_{\gamma}}{\sqrt{n}} A_{r}(X), \hat{r}(X)+\frac{u_{\gamma}}{\sqrt{n}} A_{r}(X)\right),
$$

where

$$
A_{r}(X)=\sqrt{\left(R_{1}\left(L_{2}(X)-L_{1}^{2}(X)\right)\right)^{2} \sigma_{2}^{2}\left(\hat{r}(X), v, \hat{\delta}_{r}(X)\right)},
$$

the functions $R_{1}$ and $\sigma_{2}^{2}$ are given by (12) and (24), and the statistics $\hat{r}(X)$ and $\hat{\delta}_{r}(X)$ are defined in (8) and (9).

Corollary 6. The asymptotic confidence interval with the confidence level $\gamma$ based on the estimator (9) for the unknown parameter $\delta$ has the form

$$
\left(S_{\delta_{r}}(X), T_{\delta_{r}}(X)\right)=\left(\hat{\delta}_{r}(X)-\frac{u_{\gamma}}{\sqrt{n}} A_{\delta_{r}}(X), \hat{\delta}_{r}(X)+\frac{u_{\gamma}}{\sqrt{n}} A_{\delta_{r}}(X)\right),
$$


where

$$
A_{\delta_{r}}(X)=\sqrt{s_{1}^{2}\left(\hat{r}(X), v, \hat{\delta}_{r}(X)\right)},
$$

the function $s_{1}^{2}$ is given by (27), and the statistics $\hat{r}(X)$ and $\hat{\delta}_{r}(X)$ are defined in (8) and (9).

Corollary 7. The asymptotic confidence interval with the confidence level $\gamma$ based on the estimator (10) for the unknown parameter $v$ has the form

$$
\left(S_{v}(X), T_{v}(X)\right)=\left(\hat{v}(X)-\frac{u_{\gamma}}{\sqrt{n}} A_{v}(X), \hat{v}(X)+\frac{u_{\gamma}}{\sqrt{n}} A_{v}(X)\right),
$$

where

$$
A_{v}(X)=\sqrt{\left(V_{1}\left(L_{3}(X)-3 L_{1}(X) L_{2}(X)+2 L_{1}^{3}(X)\right)\right)^{2} \sigma_{3}^{2}\left(r, \hat{v}(X), \hat{\delta}_{v}(X)\right)},
$$

the functions $V_{1}$ and $\sigma_{3}^{2}$ are given by (15) and (29), and the statistics $\hat{v}(X)$ and $\hat{\delta}_{v}(X)$ are defined in (10) and (11).

Corollary 8. The asymptotic confidence interval with the confidence level $\gamma$ based on the estimator (11) for the unknown parameter $\delta$ has the form

$$
\left(S_{\delta_{v}}(X), T_{\delta_{v}}(X)\right)=\left(\hat{\delta}_{v}(X)-\frac{u_{\gamma}}{\sqrt{n}} A_{\delta_{v}}(X), \hat{\delta}_{v}(X)+\frac{u_{\gamma}}{\sqrt{n}} A_{\delta_{v}}(X)\right),
$$

where

$$
A_{\delta_{v}}(X)=\sqrt{s_{2}^{2}\left(r, \hat{v}(X), \hat{\delta}_{v}(X)\right)},
$$

the function $s_{2}^{2}$ is given by (33), and the statistics $\hat{v}(X)$ and $\hat{\delta}_{v}(X)$ are defined in (10) and (11).

Let us illustrate the results of Corollaries $5-8$, using an example of a model sample from a gamma-exponential distribution with the given parameters $r, v, s, t$ and $\delta$. The confidence level is $\gamma=0.95$.

Tables 1-4 show the values of the estimates (8) and (9) for the parameters $r$ and $\delta$ and the estimates (10) and (11) of the parameters $v$ and $\delta$ with the corresponding asymptotic confidence intervals obtained from the sample size $n$.

Tables 5 and 6 show the proportions of the true values of parameters that fall into the asymptotic confidence intervals for 1000 runs for the sample size $n$.

Table 1. Values of the estimates of the parameters $r$ and $\delta$ with the corresponding asymptotic confidence intervals for the true values of the parameters $r=0.4, v=1.7, s=0.7, t=1.5, \delta=1$.

\begin{tabular}{ccccccc}
\hline & $\hat{r}(X)$ & $S_{r}(X)$ & $T_{r}(X)$ & $\hat{\delta}_{r}(X)$ & $S_{\delta_{r}}(X)$ & $T_{\delta_{r}}(X)$ \\
\hline$n=100$ & 0.517 & 0.344 & 0.690 & 0.782 & 0.645 & 0.919 \\
$n=1000$ & 0.391 & 0.332 & 0.449 & 0.982 & 0.924 & 1.041 \\
$n=10,000$ & 0.399 & 0.381 & 0.418 & 0.994 & 0.975 & 1.012 \\
\hline
\end{tabular}

Table 2. Values of the estimates of the parameters $v$ and $\delta$ with the corresponding asymptotic confidence intervals for the true values of the parameters $r=0.4, v=1.7, s=0.7, t=1.5, \delta=1$.

\begin{tabular}{ccccccc}
\hline & $\hat{v}(X)$ & $S_{v}(X)$ & $T_{v}(X)$ & $\hat{\delta}_{v}(X)$ & $S_{\delta_{v}}(X)$ & $T_{\delta_{v}}(X)$ \\
\hline$n=100$ & 1.048 & -0.313 & 2.409 & 0.702 & 0.207 & 1.197 \\
$n=1000$ & 1.725 & 0.977 & 2.474 & 0.981 & 0.847 & 1.115 \\
$n=10,000$ & 1.718 & 1.480 & 1.956 & 0.997 & 0.953 & 1.040 \\
\hline
\end{tabular}


Table 3. Values of the estimates of the parameters $r$ and $\delta$ with the corresponding asymptotic confidence intervals for the true values of the parameters $r=0.5, v=0.7, s=1.5, t=0.6, \delta=1$.

\begin{tabular}{ccccccc}
\hline & $\hat{r}(\boldsymbol{X})$ & $S_{r}(\boldsymbol{X})$ & $T_{r}(\boldsymbol{X})$ & $\hat{\delta}_{r}(\boldsymbol{X})$ & $S_{\delta_{r}}(\boldsymbol{X})$ & $T_{\delta_{r}}(\boldsymbol{X})$ \\
\hline$n=100$ & 0.840 & -0.637 & 2.318 & 0.827 & 0.440 & 1.214 \\
$n=1000$ & 0.445 & -0.373 & 1.264 & 1.057 & 0.914 & 1.199 \\
$n=10,000$ & 0.507 & 0.279 & 0.735 & 1.064 & 1.018 & 1.110 \\
\hline
\end{tabular}

Table 4. Values of the estimates of the parameters $v$ and $\delta$ with the corresponding asymptotic confidence intervals for the true values of the parameters $r=0.5, v=0.7, s=1.5, t=0.6, \delta=1$.

\begin{tabular}{ccccccc}
\hline & $\hat{v}(X)$ & $S_{v}(X)$ & $T_{v}(X)$ & $\hat{\delta}_{v}(X)$ & $S_{\delta_{v}}(X)$ & $T_{\delta_{v}}(X)$ \\
\hline$n=100$ & 0.668 & 0.190 & 1.147 & 0.902 & -0.688 & 2.493 \\
$n=1000$ & 0.713 & 0.555 & 0.871 & 1.016 & 0.499 & 1.533 \\
$n=10,000$ & 0.700 & 0.652 & 0.749 & 1.062 & 0.889 & 1.234 \\
\hline
\end{tabular}

Table 5. Proportions of true parameter values falling into asymptotic confidence intervals for 1000 runs for the sample size $n$ with true parameter values $r=0.4, v=1.7, s=0.7, t=1.5, \delta=1$.

\begin{tabular}{ccccc}
\hline & $\boldsymbol{r}$ & $\boldsymbol{\delta}_{\boldsymbol{r}}$ & $\boldsymbol{v}$ & $\delta_{\boldsymbol{v}}$ \\
\hline$n=100$ & 0.954 & 0.926 & 0.849 & 0.878 \\
$n=1000$ & 0.926 & 0.918 & 0.969 & 0.897 \\
$n=10,000$ & 0.920 & 0.905 & 0.977 & 0.946 \\
\hline
\end{tabular}

Table 6. Proportions of true parameter values falling into asymptotic confidence intervals for 1000 runs for the sample size $n$ with true parameter values $r=0.5, v=0.7, s=1.5, t=0.6, \delta=1$.

\begin{tabular}{ccccc}
\hline & $r$ & $\delta_{r}$ & $v$ & $\delta_{v}$ \\
\hline$n=100$ & 0.510 & 0.480 & 0.993 & 0.976 \\
$n=1000$ & 0.772 & 0.738 & 0.990 & 0.996 \\
$n=10,000$ & 0.973 & 0.916 & 0.993 & 0.999 \\
\hline
\end{tabular}

\section{Discussion}

The paper considers estimates and asymptotic confidence intervals for the parameters of the gamma-exponential distribution, represented as a scale mixture of generalized gamma distributions. Distributions from the gamma class have a rich history of modeling applications in many areas of knowledge. Back in the second half of the 19th century, the Rayleigh distribution was used to describe the resulting amplitude in the problem of summation of oscillations with random phases, and the Maxwell-Boltzmann distribution was used to statistically describe the behavior of the parameters of ideal gas particles. In the 1920s, the Italian economist L. Amoroso in the study of dynamical equilibrium theory proposed a type of distribution, which is now known as the generalized gamma distribution. The use of the generalized gamma distribution and its special cases for describing various models is still relevant. For example, in [9-12], it is proposed to use distributions from the gamma class in problems of processing radar signals and images, for studying the strength of materials and reliability of equipment, as well as for estimating the concentration of $\mathrm{NO}_{2}$ in industrial areas and studying the remission period of cancer patients. A number of other examples can be cited. The gamma-exponential distribution considered in the paper generalizes the Amoroso distribution. Therefore, it can be argued that the results of the article will be in demand when studying various models that describe real processes using distributions with a non-negative unbounded support. 
Author Contributions: Conceptualization, A.K. and O.S.; methodology, A.K. and O.S.; formal analysis, A.K. and O.S.; investigation, A.K. and O.S.; writing-original draft preparation, A.K. and O.S.; writing-review and editing, A.K. and O.S.; supervision, A.K. and O.S.; funding acquisition, O.S. All authors have read and agreed to the published version of the manuscript.

Funding: This research was supported by the Ministry of Science and Higher Education of the Russian Federation, project No. 075-15-2020-799.

Conflicts of Interest: The authors declare no conflict of interest.

\section{References}

1. Kudryavtsev, A.A. On the representation of gamma-exponential and generalized negative binomial distributions. Inform. Appl. $2019,13,78-82$.

2. Kudryavtsev, A.A.; Titova, A.I. Gamma-exponential function in Bayesian queueing models. Inform. Appl. 2017, 11, 104-108.

3. Le Roy, É. Sur les séries divergentes et les fonctions définies par un développement de Taylor. Ann. Fac. Sci. Toulouse 1900, 2, 317-384. [CrossRef]

4. Srivastava, H.M.; Tomovski, Ž. Fractional calculus with an integral operator containing a generalized Mittag-Leffler function in the kernel. Appl. Math. Comput. 2009, 211, 198-210. [CrossRef]

5. Gorenlo, R.; Kilbas, A.A.; Mainardi, F.; Rogosin, S.V. Mittag-Leffler Functions, Related Topics and Applications; Springer: Berlin, Germany, 2014.

6. Kudryavtsev, A.A. Bayesian balance models. Inform. Appl. 2018, 12, 18-27.

7. Amoroso, L. Ricerche intorno alla curva dei redditi. Ann. Mat. Pura Appl. 1925, 21, 123-159. [CrossRef]

8. Stacy, E.W. A Generalization of the Gamma Distribution. Ann. Math. Stat. 1962, 33, 1187-1192. [CrossRef]

9. Gao, G.; Ouyang, K.; Luo, Y.; Liang, S.; Zhou, S. Scheme of Parameter Estimation for Generalized Gamma Distribution and Its Application to Ship Detection in SAR Images. IEEE Trans. Geosci. Remote Sens. 2017, 55, 1812-1832. [CrossRef]

10. Zhou, Y.; Zhu, H. Image Segmentation Using a Trimmed Likelihood Estimator in the Asymmetric Mixture Model Based on Generalized Gamma and Gaussian Distributions. Math. Probl. Eng. 2018, 2018, 3468967. [CrossRef]

11. Iriarte, Y.A.; Varela, H.; Gómez, H.J.; Gómez, H.W. A Gamma-Type Distribution with Applications. Symmetry 2020, 12 , 870. [CrossRef]

12. Rivera, P.A.; Barranco-Chamorro, I.; Gallardo, D.I.; Gómez, H.W. Scale Mixture of Rayleigh Distribution. Mathematics 2020, 8, 1842. [CrossRef]

13. Lancaster, H.O. Expectations of Life: A Study in the Demography, Statistics, and History of World Mortality; Springer: New York, NY, USA, 1990

14. Bakshi, V.U.; Bakshi, U.A. Basics of Electrical Engineering; Technical Publications: Pune, India, 2008.

15. Bocharov, P.P.; DApice, C.; Pechinkin, A.V.; Salerno, S. Queueing Theory. Series "Modern Probability and Statistics"; VSP Publishing: Utrecht, The Netherlands, 2003.

16. Bostrom, A.; French, S.P.; Gottlieb, S.J. Risk Assessment, Modeling and Decision Support; Springer: Berlin, Germany, 2008.

17. Rausand, M.; Hoyland, A. System Reliability Theory: Models, Statistical Methods, and Applications; John Wiley \& Sons: Hoboken, NJ, USA, 2003.

18. Kudryavtsev, A.A.; Shestakov, O.V. Method of logarithmic moments for estimating the gamma-exponential distribution parameters. Inform. Appl. 2020, 14, 49-54.

19. Serfling, R.J. Approximation Theorems of Mathematical Statistics; John Wiley \& Sons, Inc.: New York, NY, USA, 2002. 